\title{
ANALYSIS OF THE FATIGUE STRENGTH UNDER TWO LOAD LEVELS OF A STAINLESS STEEL BASED ON ENERGY DISSIPATION
}

\author{
B. Atzori, G. Meneghetti and M. Ricotta \\ University of Padova, Department of Mechanical Engineering, 35131 Padova, Italy
}

\begin{abstract}
In this paper the fatigue behaviour of a stainless steel AISI 304L is analysed. In the first part of the work the results obtained under constant amplitude fatigue are presented and synthesised in terms of both stress amplitude and energy released to the surroundings as heat by a unit volume of material per cycle, $Q$. Then some specimens have been fatigued in variable amplitude, two different load level tests: the first level was set higher while the second was lower than the constant amplitude fatigue limit. The $Q$ values, evaluated during the second part of the fatigue test, have been compared with those calculated under constant amplitude fatigue at the same load level. The comparison allowed us to notice that the $Q$ parameter is sensitive to the fatigue damage accumulated by the material during the first part of the fatigue test.
\end{abstract}

\section{Introduction}

The evaluation of fatigue limit of materials based on the experimental measurements of thermal increments is an experimental procedure well documented in the literature [1-3]. Recently one of the Authors suggested to adopt the energy released to the surroundings as heat by a unit volume of material per cycle, $Q$, as a fatigue damage indicator [4]. In view of this, a particular strategy was conceived in order to derive the $Q$ parameter from measurements of the material surface temperature. Parameter $Q$ was able to correlate the fatigue strength of smooth and notched specimens made of stainless steel, fatigued under constant amplitude stress. To the authors' knowledge, the material behaviour in terms of $Q$ in the case of variable amplitude fatigue load was never investigated. In this paper two load level fatigue tests were carried out in order to investigate the sensitivity of $Q$ parameter as damage indicator in the case of variable amplitude fatigue.

\section{Theoretical model to estimate the $Q$ parameter}

The experimental technique used to evaluate the $Q$ parameter is based on a theoretical model presented elsewhere [4], so that only the main features will be presented here.

Let us consider a control volume $\mathrm{dV}$ of material under fatigue loading conditions, as shown in Figure 1.

This is an Open Access article distributed under the terms of the Creative Commons Attribution-Noncommercial License 3.0, which permits unrestricted use, distribution, and reproduction in any noncommercial medium, provided the original work is properly cited. 


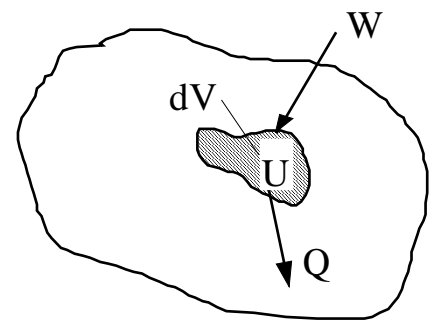

Fig. 1. First law of thermodynamics applied to a control volume of material undergoing a fatigue test.

The first law of thermodynamics applied to the control can be written in terms of power as:

$$
\mathrm{W} \cdot \mathrm{dV}=\left(\mathrm{H}_{\mathrm{cd}}+\mathrm{H}_{\mathrm{cv}}+\mathrm{H}_{\mathrm{ir}}\right) \cdot \mathrm{dV}+\left(\rho \cdot \mathrm{c} \cdot \frac{\partial \mathrm{T}}{\partial \mathrm{t}}+\dot{\mathrm{E}}_{\mathrm{p}}\right) \cdot \mathrm{dV}
$$

where $\mathrm{W}$ is the expended mechanical power in a unit volume; $\mathrm{H}_{\mathrm{cd}}, \mathrm{H}_{\mathrm{cv}}, \mathrm{H}_{\mathrm{ir}}$ represent the thermal power dissipated in a unit volume due to conduction, convection and radiation, respectively; the last term in the second member is the rate of variation of the internal energy, which is related to the material density $\rho$, the specific heat $c$, the time variation of the temperature $T$ and to the time variation of energy absorbed by the material $\dot{\mathrm{E}}_{\mathrm{p}}$. The term $\dot{\mathrm{E}}_{\mathrm{p}}$ represents the rate of accumulation of plastic hysteresis energy, i.e. fatigue damage.

Usually the surface temperature of material rapidly increases during the first part of fatigue test and then reaches a stationary value which depends on the applied stress level $[3,4]$. In steady state conditions eq (1) becomes

$$
\mathrm{W}=\left(\mathrm{H}_{\mathrm{cd}}+\mathrm{H}_{\mathrm{cv}}+\mathrm{H}_{\mathrm{ir}}\right)+\dot{\mathrm{E}}_{\mathrm{p}}
$$

By considering a sudden stop of fatigue test, the terms $\mathrm{W}$ and $\dot{\mathrm{E}}_{\mathrm{p}}$ become zero and then from eq. (1):

$$
\rho \cdot c \cdot \frac{\partial \mathrm{T}}{\partial \mathrm{t}}=-\left(\mathrm{H}_{\mathrm{cd}}+\mathrm{H}_{\mathrm{cv}}+\mathrm{H}_{\mathrm{ir}}\right)
$$

is possible to evaluate the thermal power $\mathrm{H}$ dissipated in steady state conditions (eq.(2)) by measuring the time derivative of temperature (see eq.(3)). Finally, the energy released to the surroundings as heat by a unit volume of material per cycle, $Q$, can be calculated as:

$$
\mathrm{Q}=\frac{\mathrm{H}}{\mathrm{f}}
$$

where $f$ is the test frequency.

\section{Material, specimen geometry and test procedure}

The experimental tests were conduced on specimens prepared from 6-mm-thick AISI 304L stainless steel sheets. The specimen geometries used for static and fatigue tests are shown in Figure $2 \mathrm{a}$ and Figure 2b, respectively.

Tests were carried out at room temperature on a Schenck Hydropuls PSA 100 servo-hydraulic test machine, equipped with a $100 \mathrm{kN}$ load cell. The static behaviour was investigated by means of tensile tests under displacement control with a crosshead speed equal to $2 \mathrm{~mm} / \mathrm{min}$. During the static test the axial strain was measured by means of a MTS extensometer having a gauge length of 25 $\mathrm{mm}$. The fatigue tests were carried out under load control, with a sinusoidal wave, nominal load ratio 
$\mathrm{R}\left(\mathrm{R}=\sigma_{\min } / \sigma_{\max }\right)$ equal to -1 and a test frequency variable in the range of $2-36 \mathrm{~Hz}$ depending on the applied stress level. To investigate the material fatigue behaviour, constant amplitude fatigue tests were carried out up to the specimen failure. Concerning the two load level fatigue tests, some specimens were fatigued at a stress level higher than the fatigue limit for a significant fraction of fatigue life. Then the stress level was decreased lower than the fatigue limit and kept constant up to 10 millions of cycles or specimen failure.

Temperature increments were monitored by means of an AGEMA THV 900 LW/ST infrared camera able to detect infrared radiation in the range of wave lengths between 8 and $12 \mathrm{~mm}$ with a resolution of $0.1{ }^{\circ} \mathrm{C}$. The thermal images were post processed by using the dedicated software AGEMA research 2.1.
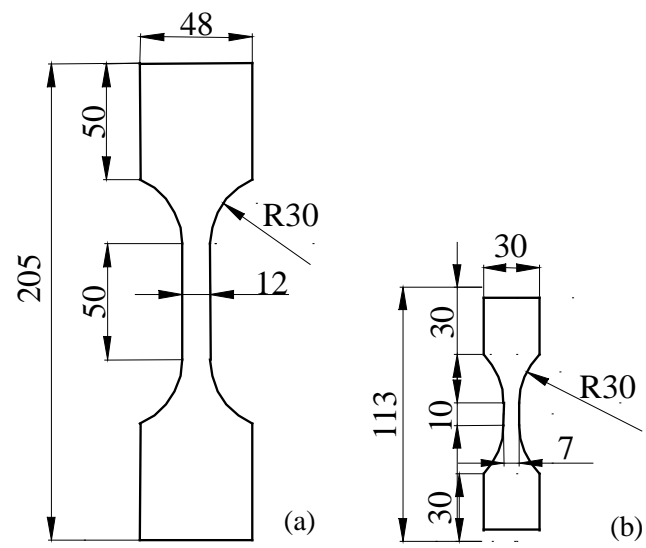

Fig. 2. Specimen geometry for static (a) and fatigue (b) tests.

\section{Experimental results}

In order to characterise the material static behaviour, five tests were carried out. The mean value of elastic modulus $\mathrm{E}$, engineering tensile strength $\sigma_{\mathrm{R}}$, proof strength $\sigma_{\mathrm{p} 0,2}$ and true fracture strain A\% are summarised in Table 1. By means of an electrolytic etching (stainless steel anode and cathode, voltage $1.2 \mathrm{~V}$, current $0.2 \mathrm{~A}$ ) on a $60 \%$ nitric acid solution the microstructure was analysed. A typical example is shown in Figure 3: the white matrix represents the austenitic phase while the dark zones inside the grains are ferrite, which represents the $1 \%$ of the volume.

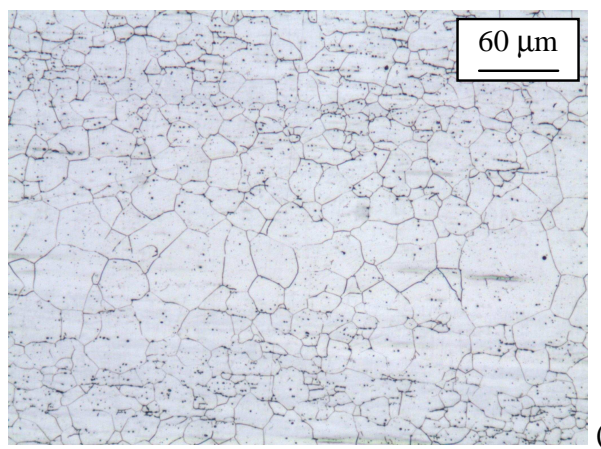

(a)

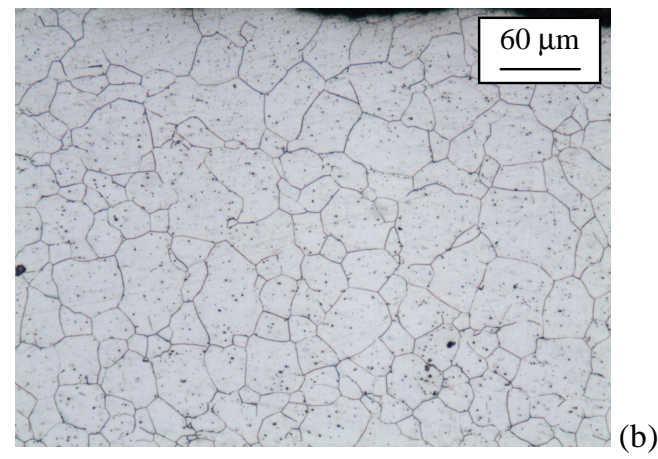

Fig. 3. Example of microstructure observed in the cross section: mid-thickness (a) and below surface (b)

The grain size was assessed according to ASTM E112 standard [5]. Two specimens were analysed and three measurements were conduced on each of them: in particular, two measurements 
were performed in the cross-section (one in the mid thickness and one below specimen's surface) and one on the surface of the sheet. The mean values are listed in Table 2. One can note that the grains are bigger in the external surface, as it can be expected in the case of rolling sheets. Finally, measurements of Vickers micro-hardness (applied load $0.2 \mathrm{~kg}$ ) were carried out. Table 3 shows the results obtained in the middle of the thickness and at $0.2 \mathrm{~mm}$ below the surface.

Table 1. Material properties of AISI 304L stainless steel

\begin{tabular}{cccc}
\hline $\mathrm{E}[\mathrm{MPa}]$ & $\sigma_{\mathrm{p} 0,2}[\mathrm{MPa}]$ & $\sigma_{\mathrm{R}}[\mathrm{MPa}]$ & $\mathrm{A} \%$ \\
\hline 194750 & 315 & 699 & $59 \%$ \\
\hline
\end{tabular}

Table 2. Experimentally measured grain size.

\begin{tabular}{lcc}
\hline & Specimen $1[\mu \mathrm{m}]$ & Specimen $2[\mu \mathrm{m}]$ \\
\hline Below surface & 37 & 27 \\
Mid-thickness & 30 & 27 \\
Surface & 41 & 35 \\
\hline
\end{tabular}

Table 3. Measured values of Vickers micro-hardness (applied load $0.2 \mathrm{~kg}$ ).

\begin{tabular}{lcc}
\hline & Specimen 1 & Specimen 2 \\
\hline \multirow{3}{*}{ Below surface } & 175 & 155 \\
& 163 & 157 \\
& 151 & 151 \\
\hline \multirow{3}{*}{ Mid-thickness } & 169 & 170 \\
& 162 & 163 \\
& 162 & 167 \\
\hline
\end{tabular}

\subsection{Synthesis of experimental data in terms of stress amplitude}

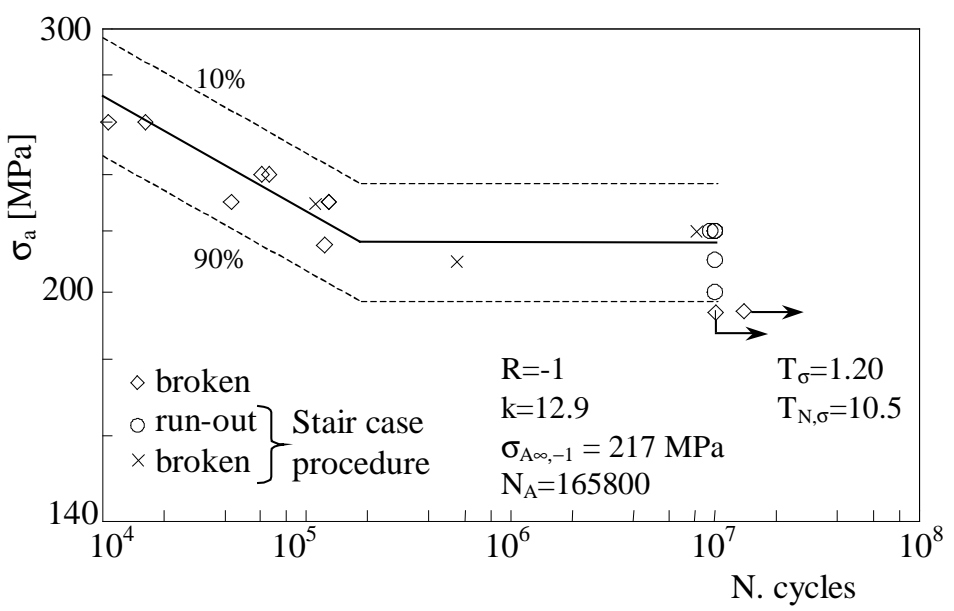

Fig. 4. Wöhler curve and 10\%-90\% scatter band of the AISI $304 \mathrm{~L}$ stainless steel. 
The material fatigue limit was evaluated according to a shortened stair-case procedure according to Dixon's rule [6], which involved 7 specimens. Tests were stopped after 10 million cycles. As a results, the fatigue limit in terms of stress amplitude $\sigma_{\mathrm{A} \infty,-1}$ resulted equal to $217 \mathrm{MPa}$.

The Wöhler curve, shown in Figure 4, was evaluated via statistical analysis of the available fatigue data, by assuming log-normal distribution of the number of cycles to failure. In the same figure the fatigue limit $\sigma_{\mathrm{A} \infty,-1}$, the 10-90\% scatter band, the value of the inverse slope $k$ of Wöhler curve, the scatter index $\mathrm{T}_{\sigma}\left(\mathrm{T}_{\sigma}=\sigma_{\mathrm{A} \infty,-1,10 \%} / \sigma_{\mathrm{A} \propto,-1,90 \%}\right)$, the scatter index $\mathrm{T}_{\mathrm{N}, \sigma}\left(\mathrm{T}_{\mathrm{N}, \sigma}=\mathrm{T}_{\sigma}{ }^{\mathrm{k}}\right)$ and the number of cycles $\mathrm{N}_{\mathrm{A}}$, which corresponds to the knee point of the curve, are listed.

\subsection{Synthesis of experimental data in terms $Q$ parameter}

As already discussed, the evaluation of $Q$ parameter is based on the measurement of the cooling rate just after a sudden interruption of the fatigue test, according to equations (3) and (4). The material density, experimentally measured by using Archimedes method and a Sartorius 1801 balance, with a resolution of $10^{-7} \mathrm{~kg}$, was $7940 \mathrm{~kg} / \mathrm{m}^{3}$. By using a calorimeter, the specific heat $c$ was determined equal to $507 \mathrm{~J} \cdot \mathrm{kg}^{-1} \cdot \mathrm{K}^{-1}$.

The cooling rate after a sudden interruption of the fatigue test was measured by using the infrared camera and by considering the maximum value of the temperature inside on area encompassing the reduced section of the specimen. The maximum sampling frequency of the thermal images allowed by the available measuring system was $7 \mathrm{~Hz}$. Figure 5a shows an example of an infrared image: the rectangle identifies the area where the maximum surface temperature was detected. A typical example of the recorded temperature trend is plotted in Figure $5 \mathrm{~b}: \mathrm{t}_{0}$ indicates the time when the fatigue test was stopped while in the y-axis the temperature variation with respect to that stabilized before the test stop is shown. After evaluating the cooling gradient, $Q$ was derived according to equations (3) and (4).
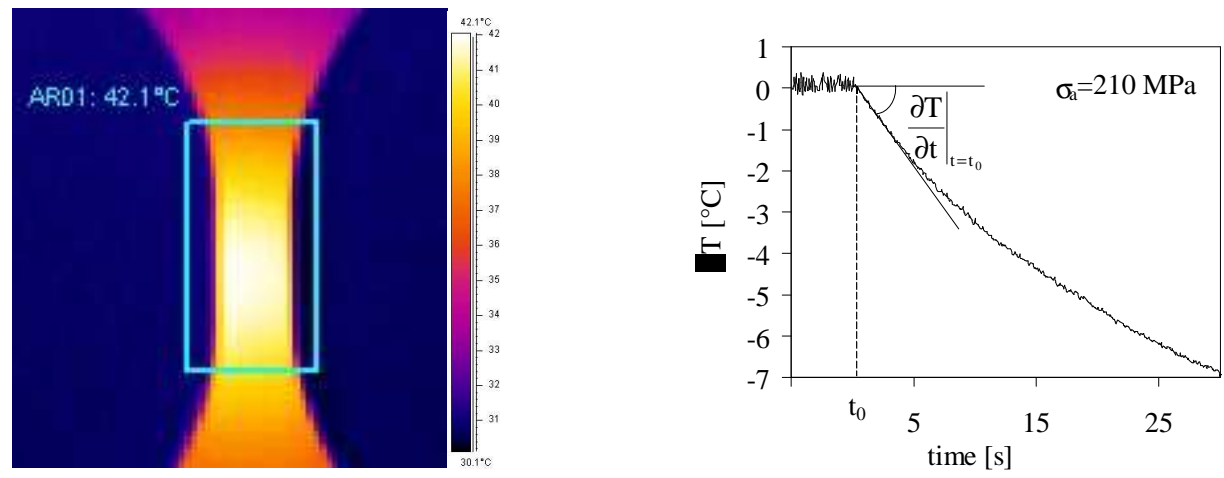

Fig. 5. Example of control area surrounding the specimen where the maximum temperature was analysed (a) and typical maximum temperature signal measured after a sudden interruption of fatigue test $(\mathrm{b})\left(\sigma_{\mathrm{a}}=210 \mathrm{MPa}\right.$, run out).

In order to evaluate the evolution of $Q$ parameter, each fatigue test was interrupted several times. Figure 6 shows the $Q$ values plotted versus the number of cycles normalised with respect to the number of cycles to failure or, in the case of run-out specimens, with respect to 10 millions. It can be noted that the value of $Q$ reached a constant value after about $50 \%$ of the total fatigue life. Moreover, the plotted curves show as soon as the stress amplitude is increased above the fatigue limit $\left(\sigma_{\mathrm{a}}>220\right.$ $\mathrm{MPa}$ ) then the stabilised values of $Q$ increase of a factor 7 (from $\approx 100 \mathrm{~kJ} /\left(\mathrm{m}^{3} \cdot\right.$ cycle) to $\approx 700$ $\mathrm{kJ} /\left(\mathrm{m}^{3} \cdot\right.$ cycle $\left.)\right)$.

The fatigue data were analysed in terms of the stabilised energy parameter found during each fatigue test, by assuming a log-normal distribution of the number of cycles to failure, according to the following equation: 


$$
\mathrm{Q}^{\mathrm{k}} \cdot \mathrm{N}=\cos \mathrm{t}
$$

where $\mathrm{N}$ represents the number of cycles to failure and $\mathrm{k}$ is the inverse slope of the new fatigue curve.

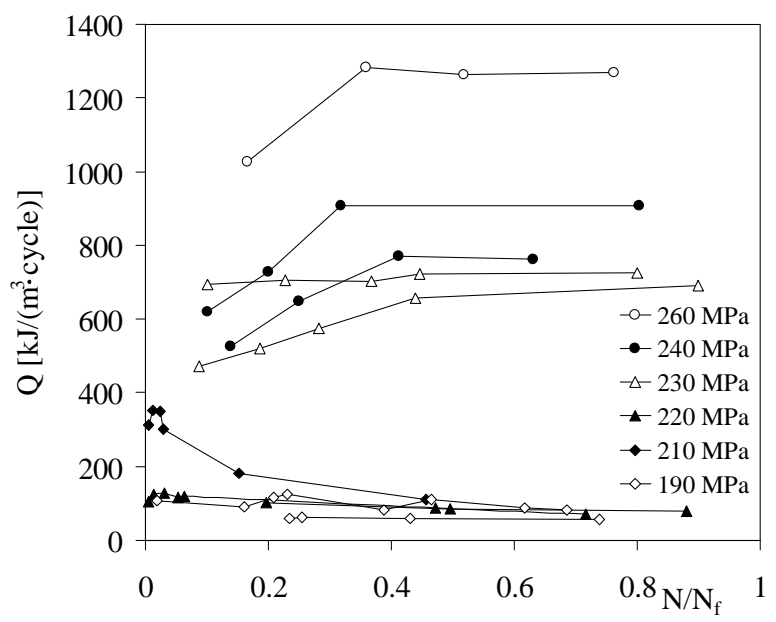

Fig.6. Evolution of the specific energy loss versus the normalised fatigue life.

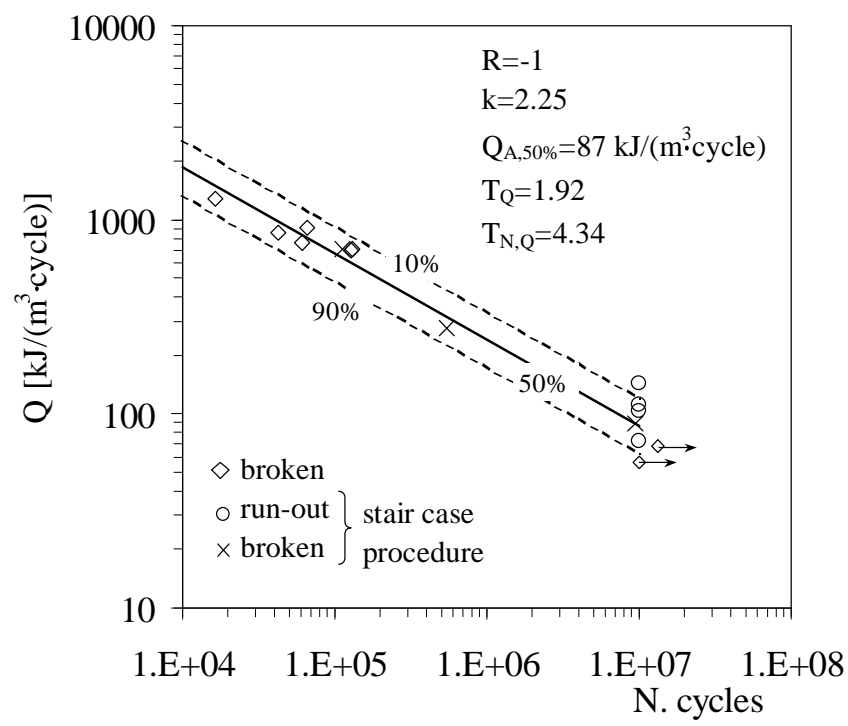

Fig. 7. Fatigue data synthesised in terms of specific energy loss $Q$ for the AISI $304 \mathrm{~L}$ stainless steel.

Figure 7 shows the fatigue data analysed according to eq. (5), with the 10-90\% scatter band, the reference $Q$ value evaluated at 10 millions of cycles, the value of exponent $k$, the scatter index $\mathrm{T}_{\mathrm{Q}}$ $\left(\mathrm{T}_{\mathrm{Q}}=\mathrm{Q}_{\mathrm{A}, 10 \%} / \mathrm{Q}_{\mathrm{A}, 90 \%}\right)$ and the scatter index $\mathrm{T}_{\mathrm{N}, \mathrm{Q}}\left(\mathrm{T}_{\mathrm{N}, \mathrm{Q}}=\mathrm{T}_{\mathrm{Q}}{ }^{\mathrm{k}}\right)$. The experimental data can be synthesised in a unique scatter band characterised by a constant slope $k$ from $10^{4}$ to $10^{7}$ cycles. It is interesting to note that the scatter index $T_{N, Q}$ results by far lower than $T_{N, \sigma}$ shown in Figure 4 .

\section{Two load level fatigue tests}


With the aim to evaluate the sensitivity of the $Q$ parameter to prior fatigue damage, some specimens were fatigued in variable amplitude, two different load level tests: the first level was chosen higher $\left(\sigma_{\mathrm{a}}=230 \mathrm{MPa}\right)$ than the material fatigue limit $\left(\sigma_{\mathrm{A}, \infty}=217 \mathrm{MPa}\right)$ and it was applied for a significant fraction of fatigue life (ranging from 80 to $86 \%$ ) as evaluated according to the $Q-N$ curve, previously obtained by means of constant amplitude fatigue tests (see Figure 7). The second load level $\left(\sigma_{\mathrm{a}}=190\right.$ $\mathrm{MPa}$ ) was lower than the fatigue limit. It should be noted that the two-load level fatigue tests presented in this paper are different from those presented in [4], where both levels were higher than the constant amplitude fatigue limit. The $Q$ values, evaluated during the second step of the fatigue test, have been compared with those measured during some tests carried out under constant amplitude fatigue at the same load level on undamaged specimens.

The results of the two load level fatigue tests are shown in Figure 8 in terms of stress amplitude. It can be noted that only two of four specimens failed during the second level fatigue test. The same results were re-analysed on the basis of the energy parameter $Q$ and plotted in Figure 9: in the case of specimens that failed, the $Q$ values measured during the second block of cycles were definitely higher than those observed during constant amplitude fatigue tests at the same stress level on the undamaged material. On the contrary, specimens 3 and 4 exceeded 10 millions of cycles once loaded at the second stress level. In fact for these specimens the $Q$ values measured during the second block of cycles were very close to those detected during constant amplitude fatigue tests at $190 \mathrm{MPa}$ on undamaged material.

Then, as far as the experimental data analysed in the present paper are concerned, it can be concluded that the parameter $\mathrm{Q}$ is sensitive to the fatigue damage accumulated prior to the second block of fatigue cycles.

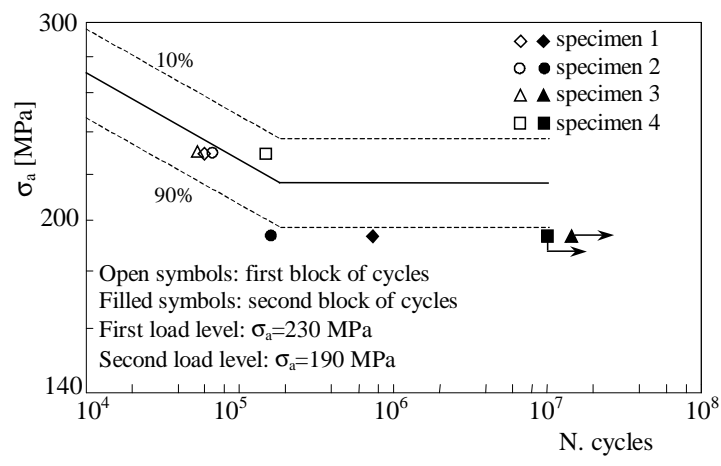

Fig. 8. Results of two load level fatigue tests in terms of stress. Scatter band is that reported in Figure 4.

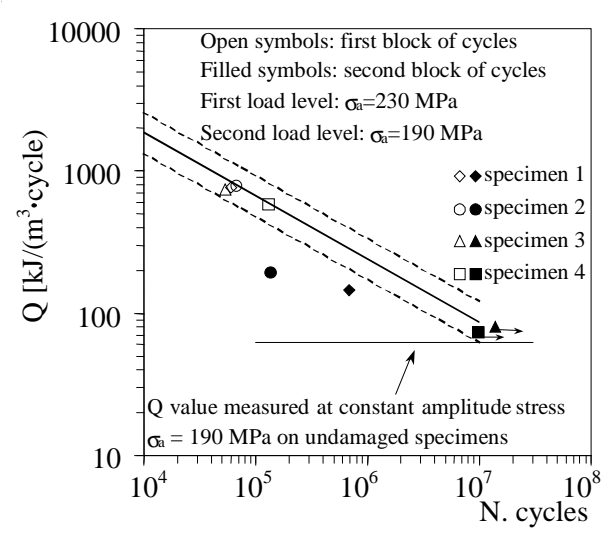

Fig. 9. Results of two load level fatigue tests in terms of Q. The scatter band is that reported in Figure 7.

Finally, by considering only the two specimens that failed, the damage $D$ was evaluated on the basis of the $Q-N$ curve shown by Figure 7, according to the Miner's rule (eq. 6):

$$
\mathrm{D}=\sum_{\mathrm{i}=1}^{2} \frac{\mathrm{n}_{\mathrm{i}}}{\mathrm{N}_{\mathrm{i}}}=\frac{\mathrm{n}_{1}}{\mathrm{~N}_{1}}+\frac{\mathrm{n}_{2}}{\mathrm{~N}_{2}}
$$

where $n_{i}$ is the number of cycles carried out at $\sigma_{a, i}$ stress amplitude and $N_{i}$ represents the corresponding number of cycles to failure. Figure 10 compares the experimental results obtained for specimen 1 and 2 with Miner's hypothesis $(\mathrm{D}=1)$ : the horizontal and the vertical axis represent the fraction of fatigue life spent during the first block $\left(\mathrm{n}_{1} / \mathrm{N}_{1}\right)$ and during the second block $\left(\mathrm{n}_{2} / \mathrm{N}_{2}\right)$ of load, respectively. The comparison allowed us to notice that the Miner's hypothesis, based on the $Q$ $N$ curve, is in good agreement with the experimental results. 


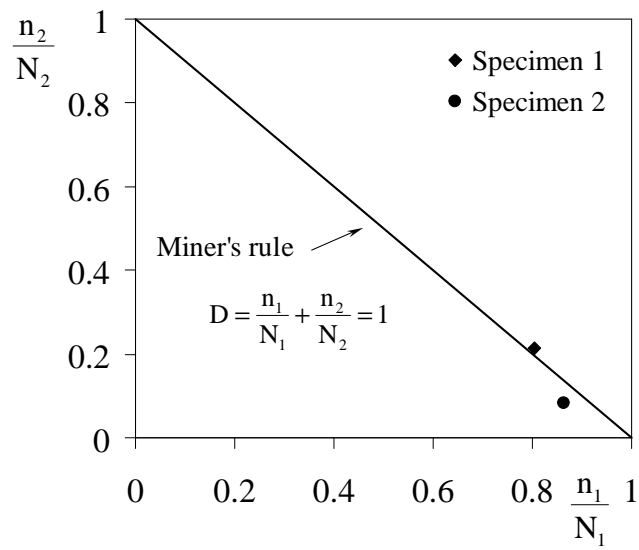

Fig. 10. Comparison between the experimental data and Miner's rule applied in terms of $Q$.

\section{Conclusions}

In this paper the fatigue behaviour of a stainless steel AISI $304 \mathrm{~L}$ was analysed in terms of energy released to the surroundings as heat by a unit volume of material per cycle, $Q$. After evaluating the material constant amplitude fatigue limit under completely reversed axial stress, the fatigue data were analysed in terms of both stress amplitude and energy parameter $Q$, according to [4]. Then some specimens were fatigued in variable amplitude, two load levels to investigate the sensitivity of the $Q$ parameter to prior fatigue damage.

The main results can be summarised as follows:

- the Wöhler curve of material generated from constant amplitude fatigue tests presents a knee point at 165800 cycles;

- the average curve which synthesises the fatigue data in terms of the energy parameter $Q$ presents a constant slope from $10^{4}$ to $10^{7}$ cycles;

- the data dispersion in terms of fatigue lives is reduced by a factor greater than two if the experimental data are processed by using the energy parameter $Q$ rather than the applied stress amplitude;

- the observed energy parameter $Q$ increases by a factor of about seven if specimens are tested just $13 \mathrm{MPa}$ above the fatigue limit with respect to the values measured at or below the fatigue limit itself;

- it can be concluded that the parameter $Q$ is a fatigue indicator sensitive to the damage accumulated during the prior load history;

- Miner's hypothesis applied on the basis of the $Q-N$ curve is in good agreement with the experimental data available.

As a final remark, additional work is needed to further support the experimental findings presented in this paper.

\section{References}

1 D. Dengel, H. Harig, Fatigue Fract Engng Mater Struct, 3:113-28, (1980)

2 M.P. Luong, Mech Mater, 28:155-63, (1988)

3 G. La Rosa, A. Risitano, Int J Fatigue, 22, 65-73, (2000).

4 G. Meneghetti, Int J Fatigue, 29, 81-94, (2007).

5 ASTM E112 - 96(2004)e2 Standard Test Methods for Determining Average Grain Size.

6 W. Dixon, F. Massey, Introduction to statistical analysis, McGraw-Hill, 1966, New York. 